\title{
Anestesia neuroaxial en trabajo de parto y cesárea en pacientes con antecedentes de mielomeningocele operado
}

\author{
Andrea González S. ${ }^{1}$, Claudio Nazar J. ${ }^{2}$, Javier Bastidas E. ${ }^{1}$, Maximiliano Zamora H. ${ }^{a}$, \\ Patricio Mellado T. ${ }^{3}$, Héctor Lacassie Q. 2 \\ ${ }^{1}$ Programa de Posgrado en Anestesiología, ${ }^{2}$ División de Anestesiología, ${ }^{a}$ Alumno de Medicina, ${ }^{3}$ Departamento de \\ Neurología. Escuela de Medicina, Pontificia Universidad Católica de Chile.
}

a Alumno, Escuela de Medicina, Pontificia Universidad Católica de Chile.

\section{RESUMEN}

Los desórdenes del desarrollo del tubo neural son el segundo mayor grupo de malformaciones congénitas conocidas y con una incidencia de 1/1000 nacidos vivos. El meningomielocele es una de las malformaciones más frecuentes. Se define como una falla en el cierre del tubo neural con herniación de meninges y elementos neurales. El embarazo en estas pacientes es complicado por las deformidades físicas y complicaciones neurológicas secundarias, pudiendo dificultar la técnica anestésica neuroaxial en el trabajo de parto y operación cesárea. Existen escasos reportes de pacientes con meningomielocele en trabajo de parto y analgesia neuroaxial. Presentamos una revisión de esta patología y las técnicas anestésicas utilizadas en el trabajo de parto y operación cesárea de pacientes con antecedente de meningomielocele, basados en un caso clínico del cual participamos.

\section{PALABRAS CLAVE: Meningomielocele, embarazo, anestesia obstétrica, anestesia epidural, trabajo de parto}

\section{SUMMARY}

Neural tube defects are the second most common type of congenital birth defects with an incidence of $1 / 1000$ newborns. Meningomyelocele is one of the most common clinical presentations. It is defined as a failed closure of the neural arch with herniation of the meninges and neural elements. Pregnancy among these patients can be complicated with physical deformity and coexisting neurological defects, which may challenge neuroaxial anesthetic technique in obstetric labor and cesarean delivery. There are few reports involving patients with meningomyelocele in obstetric labor and neuroaxial anesthesia. We discuss some key points of this disease and the anesthetic technique of choice in obstetric labor and cesarean delivery in patients with history of meningomyelocele, based on a case in which we participated.

KEY WORDS: Meningomyelocele, pregnancy, obstetrical anesthesia, epidural anesthesia, obstetric labor 


\section{INTRODUCCIÓN}

Los desórdenes del desarrollo del tubo neural son el segundo grupo de malformaciones congénitas más comunes, después de las malformaciones cardíacas. Tienen una incidencia de 1/1000 nacidos vivos (1), siendo más común en niñas que niños. El espectro de anomalías comprende desde defectos del segmento cefálico incompatibles con la vida extrauterina (como la anencefalia) hasta la espina bífida oculta (descubierta al azar en una radiografía simple pélvica) (2). La etiología del defecto del tubo neural es heterogénea y compleja, con factores ambientales y genéticos involucrados en su patogenia. Sin embargo, la causa exacta no es conocida en la mayoría de los casos. La prevalencia de defectos del tubo neural varía a través del tiempo, región y etnia (2). Una de las presentaciones más comunes de defecto del tubo neural clínicamente significativo es la espina bífida cística, siendo el mielomeningocele la forma más frecuente.

La historia de anencefalia y espina bífida familiar es el factor de riesgo más preponderante para estos defectos. Otros factores de riesgo incluyen: diabetes mellitus pregestacional y gestacional, bajo consumo de ácido fólico y uso materno de drogas anticonvulsivantes (ácido valproico y carbamazepina) (3).

Actualmente, gracias a los avances en la medicina, muchas pacientes que fallecían a temprana edad han alcanzado la edad adulta, pudiendo embarazarse durante su época fértil. El embarazo en estas pacientes es complicado por las deformidades físicas y las complicaciones neurológicas secundarias. En la actualidad, se presenta un nuevo desafío para el equipo obstétrico, especialmente en relación al manejo de la analgesia durante el trabajo de parto y en la elección de la mejor técnica anestésica para una operación cesárea en las pacientes con el antecedente de mielomeningocele, debiendo existir una precoz y adecuada comunicación entre obstetras y anestesiólogos.

El objetivo de esta publicación es realizar una revisión de la evidencia médica existente sobre mielomeningocele y sus implicancias en las técnicas anestésicas a utilizar en el trabajo de parto y operación cesárea en este tipo de pacientes, la cual fue motivada por un caso clínico atendido en la institución.

\section{Caso clínico}

Paciente de 20 años, $65 \mathrm{~kg}$ de peso y altura de $154 \mathrm{~cm}$, que cursó un embarazo fisiológico de 40 +1 semanas por fecha de última menstruación. El único antecedente que describió fue un mielomeningocele bajo L3, operado a los 10 días de vida, sin secuelas neurológicas, disfunción intestinal ni urinaria postoperatoria. A las 33 semanas de gestación fue evaluada electivamente por un anestesiólogo de nuestra institución. Destacó al examen físico una cicatriz vertical de $3 \mathrm{~cm}$ de longitud en la línea media de la región lumbosacra. El examen neurológico fue normal (con movilidad, sensibilidad y potencia muscular de las extremidades inferiores conservadas). Se solicitó una resonancia nuclear magnética (RM) dorsolumbar que se realizó a las 34 semanas de gestación, la cual demostró un cono medular en nivel L3, con un filum terminale engrosado, concluyendo alteraciones compatibles con médula anclada (Figura 1). Se explicó a la paciente los beneficios y riesgos de la anestesia neuroaxial (epidural en caso de trabajo de parto, espinal en caso de cesárea electiva), con énfasis en el mayor riesgo de punción de duramadre-aracnoides con la técnica epidural, los que entendió y aceptó. En conjunto con su obstetra tratante, se programó para parto vaginal con analgesia epidural (evolución espontánea o inducción a las 41 semanas).

La paciente ingresó a la maternidad de la institución a las $40+1$ semanas de gestación en trabajo de parto espontáneo. Al examen obstétrico se constató dinámica uterina de 2 en 10 minutos, membranas íntegras, cuello $100 \%$ borrado y $4 \mathrm{~cm}$ de dilatación cervical. El feto estaba en presentación cefálica y con latidos cardiacos fetales normales. A los 60 minutos de su ingreso, la paciente solicitó analgesia epidural.

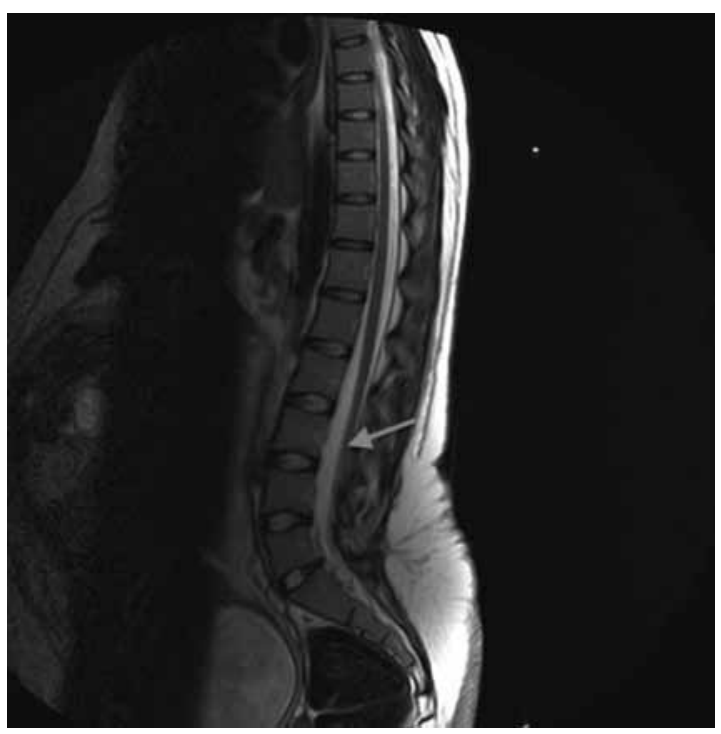

Figura 1. Resonancia magnética dorsolumbar de la paciente. Descripción: imagen en T2, corte sagital a nivel de línea media. Se observa un cono medular en nivel L3 (flecha), con un filum terminale engrosado, compatibles con el diagnóstico de médula anclada. 
Se puncionó en el espacio intervertebral L2-L3, con técnica de pérdida de resistencia con solución salina, encontrándose el espacio epidural a $5 \mathrm{~cm}$ desde la piel. Se introdujo un catéter peridural № $18 \mathrm{G}$ en dirección cefálica, fijándose a $9 \mathrm{~cm}$ en piel. Se administraron dosis sucesivas en bolos por el catéter peridural con buena respuesta analgésica.

Después de aproximadamente 8 horas de trabajo de parto, estando la paciente con dilatación cervical completa y en plano espinas cero durante más de 1 hora, el obstetra decidió realizar una operación cesárea por falla en el descenso fetal. El anestesiólogo tratante decidió utilizar el catéter epidural para anestesia de cesárea. Se inyectaron $10 \mathrm{ml}$ de lidocaína al $2 \%$ más fentanyl $50 \mu \mathrm{g}$ en un volumen total de $11 \mathrm{ml}$. Luego de 20 minutos se alcanzó un nivel anestésico sensitivo T4 y se inició la cirugía. La paciente se mantuvo hemodinámicamente estable con cohidratación con cristaloides e infusión de fenilefrina endovenosa por bomba de jeringa a $1 \mu^{*} \mathrm{~kg}^{-1 *} \mathrm{~min}^{-1}$ inicial y ajustando para una presión arterial media (PAM) $\pm 20 \%$ de la basal. Al ingresar el obstetra a la cavidad abdominal, la paciente refirió dolor por lo que se administró remifentanilo endovenoso por bomba de jeringa a $0,1 \mu \mathrm{g}^{*} \mathrm{~kg}^{-1}{ }^{*} \mathrm{~min}^{-1}$, mejorando considerablemente la analgesia. A los 16 minutos de iniciada la cirugía se extrajo un recién nacido vivo, sexo femenino, de 3750 gramos y Apgar 8 - 9. El resto de la intervención quirúrgica se completó sin incidentes. Al finalizar la cirugía se suspendió la infusión endovenosa de remifentanilo. No hubo nuevos incidentes anestésicos. La paciente fue dada de alta a su pieza a las 2 horas post cirugía con recuperación completa sensitiva y motora de extremidades inferiores y sin incidentes. Se retiró el catéter epidural íntegro y sin problemas. Al tercer día postoperatorio la paciente fue dada de alta a su hogar, asintomática para cefalea postpunción dural y sin alteraciones neurológicas, condición que se mantuvo sin cambios en los controles a los 7 y 30 días postoperatorios.

\section{DISCUSIÓN}

La espina bífida describe un grupo de condiciones que están divididas en dos: espina bífida oculta (EBO) y espina bífida cística (EBC) (4).

$E B O$ : Surge cuando las dos mitades del arco vertebral fallan en la unión en la línea media. La médula espinal y las raíces nerviosas son normales. No existe lesión externa visible y generalmente la piel que lo cubre es normal. Ésta es una condición benigna y no está asociada con ningún síntoma neurológico. Frecuentemente es sólo un hallazgo incidental en una radiografía y puede estar presente en hasta el $20 \%$ de la población (5). Comúnmente se observa en la región lumbosacra, pero también puede observarse en la región cervical.

$E B C$ : Es un término colectivo que envuelve a las dos formas más graves de espina bífida: Espina bífida con meningocele o con meningomielocele (6). La característica común es la presencia de un saco que protruye a través del defecto óseo en la columna vertebral. En pacientes con meningocele, el saco que protruye contiene meninges y líquido cefalorraquídeo (LCR), sin involucrar elementos neurológicos. La médula espinal y las raíces nerviosas espinales están en su posición normal, pero pueden ser anormales. En la espina bífida con meningomielocele, el saco que protruye tiene incorporado raíces nerviosas y médula espinal en grado variable y es usualmente una lesión abierta y expuesta.

El meningomielocele es una de las formas de presentación más frecuente de las mielodisplasias y comprende a cerca del $95 \%$ de los casos de EBC. Es usual ver un déficit neurológico bajo el nivel del defecto, comprometiendo la deambulación y provocando alteraciones en el control de vejiga e intestino. Una combinación de parálisis flácida y espástica está presente en el 20 a $40 \%$ de los casos y requerirá de un tratamiento ortopédico-quirúrgico para permitir una deambulación funcional. Estos pacientes tienen una alta incidencia de malformaciones congénitas asociadas: defectos en el sistema respiratorio, músculo-esquelético, cardiovascular; además de una alta incidencia de escoliosis (sobre el $50 \%$ ). Los pacientes con meningomielocele se asocian, prácticamente en todos los casos, con malformación Arnold Chiari tipo II (herniación de la vermis cerebelosa por el foramen magnum).

La hidrocefalia y sus complicaciones son la mayor causa de muerte en los primeros años de vida. Las mayores causas de morbilidad y mortalidad después de los tres años son la pielonefritis y la insuficiencia renal. Estudios poblacionales demuestran un aumento en la sobrevida a largo plazo, gracias al diagnóstico antenatal, cirugía precoz (incluso intrauterina), y manejo temprano y adecuado de la hidrocefalia. Actualmente la sobrevida es de un $80 \%$.

Es un consenso general que la severidad de la invalidez física de los pacientes con espina bífida que llegan a la adultez, está determinado por el grado del daño de tejido nervioso, ya sea como resultado del defecto primario o como consecuencia de la cirugía correctiva.

Un estudio retrospectivo realizado el año 2002, evaluó a los pacientes nacidos con espina bífida entre los años 1963 al 1971 y operados en los primeros días de vida. Se encontraron 117 pacientes y el $54 \%$ había muerto. Las causas más importantes de mortalidad fueron complicaciones cardiorrespiratorias, insuficiencia renal, hidrocefalia e infección del sistema nervioso central. Las lesiones bajo L3 tienen menor morbimortalidad que las sobre este nivel (7), lo que explicaría la buena evolución y el escaso compromiso neurológico de nuestra paciente. 
El embarazo en pacientes con espina bífida es complicado por la deformidad física (escoliosis, cifosis, displasia de caderas, etc.), las cirugías urinarias y neurológicas previas, las válvulas derivativas ventrículo peritoneales para controlar la hidrocefalia, el grado variable de daño renal, hipertensión arterial y una alta incidencia de alergia al látex. Esta última debido a la sensibilización al agente producto del uso crónico de sondas vesicales de látex para vaciado vesical intermitente por la vejiga neurogénica secundaria al defecto neural.

No existe regla general que considere la vía del parto de estas pacientes. Cada paciente requiere un enfoque individual. Existen reportes de partos vaginales espontáneos normales. Dependiendo del nivel de la lesión, el pujo voluntario puede estar comprometido, requiriendo grandes episiotomías o la aplicación de fórceps para el expulsivo. La deformidad esquelética pélvica y vertebral puede provocar desproporción cefalopélvica y la necesidad de cesárea (8).

Actualmente se acepta que el estándar dorado para el diagnóstico de este tipo de patologías es la resonancia magnética (RM). Dentro de sus ventajas encontramos que es capaz de mostrar el nivel exacto del defecto espinal y su extensión. Además, no está contraindicada durante el embarazo y permite evaluar la factibilidad de utilizar una técnica regional para analgesia durante un trabajo de parto o anestesia para una operación cesárea, determinando el nivel al cual puede puncionarse (9-11).

Existen muy pocos casos publicados sobre el manejo analgésico y anestésico de estas pacientes en el trabajo de parto y operación cesárea. No existen metaanálisis ni guías clínicas que permitan orientarnos mejor y con buena evidencia en la administración de la analgesia-anestesia neuroaxial de las pacientes con espina bífida. La literatura médica sólo se refiere a reportes de casos aislados o grupos pequeños de pacientes (Tabla I) (12-16). Se ha reportado uso de analgesia-anestesia epidural, caudal, espinal, bloqueo simpático paravertebral, bloqueo paracervical y uso de analgesia controlada por paciente (PCA) endovenosa en base a opioides. Todas estas técnicas han sido asociadas a numerosas limitaciones y complicaciones en pacientes con espina bífida.

En pacientes con EBO la técnica espinal o peridural no está contraindicada y generalmente no presenta mayores complicaciones. Sin embargo, es recomendable que la punción sea realizada sobre el nivel de la lesión y por un anestesiólogo con experiencia. Si se intenta colocar un catéter peridural en el nivel de la EBO, la punción dural y sus eventuales complicaciones son inevitables (17).

Es importante tener presente la posibilidad de disrafia espinal en las pacientes con EBO. Si en una paciente se presentan anormalidades neurológicas, manifestaciones cutáneas, o la EBO involucra a más de una lámina vertebral, existe una incidencia entre 35 - $87 \%$ que la médula espinal termine por debajo de L2-L3 (6).

La incidencia de síndrome de médula espinal anclada después de cirugía correctiva del defecto del tubo neural es de alrededor de un $4 \%$, pero la sintomatología de este síndrome puede ser mucho menor (18). Sin embargo, la incidencia de adhesión entre la médula espinal y la duramadre es casi del $100 \%$ cuando la paciente es evaluada con RM. El hecho de que la médula espinal y las raíces nerviosas terminen en un nivel más bajo que en los pacientes normales, incluso después de la cirugía correctiva (9), es un dato importante para el anestesiólogo, el cual debe considerar este hecho al momento de realizar la anestesia epidural, espinal y/o combinada.

\section{CONCLUSIONES}

A medida que han mejorado las técnicas radiológicas, incluyendo la RM, y que han aumentado los reportes de casos de pacientes embarazadas con espina bífida, el manejo de la analgesia para el parto y anestesia para la operación cesárea con anestesia regional ha mejorado notablemente, con menor incidencia de punción dural, complicaciones neurológicas y resultados desfavorables, logrando analgesia satisfactoria en la mayoría de los casos. Es fundamental recalcar que para lograr en estas pacientes un parto y anestesia regional seguros, deben ser evaluadas tempranamente durante el desarrollo del embarazo por un equipo multidisciplinario (obstetra, matrona, neurólogo y anestesiólogo). Es responsabilidad del equipo obstétrico conocer y entender completamente la terminología y la extensión del defecto neurológico antes de planificar la vía de parto y realizar una anestesia neuroaxial $(4,6)$. 


\section{Tabla I \\ LITERATURA MÉDICA SOBRE VÍA DEL PARTOY ANALGESIA-ANESTESIA UTILIZADA EN PACIENTES CON ESPINA BÍFIDA}

\begin{tabular}{|c|c|c|c|c|}
\hline $\begin{array}{l}\text { Reporte } \\
\text { (referencia) }\end{array}$ & Vía de parto (n) & Casos (n) & Técnica & Resultados \\
\hline $\begin{array}{l}\text { Vaagenes }^{(12)} \\
(1981)\end{array}$ & Vaginal & 1 & Peridural & $\begin{array}{l}\text { Recién nacido vivo. Peso: } 2730 \mathrm{~g} \text {. } \\
\text { Apgar } 9-10 . \text { Madre satisfecha con } \\
\text { analgesia durante expulsivo. }\end{array}$ \\
\hline $\begin{array}{l}\text { McGrady }{ }^{(13)} \\
(1988)\end{array}$ & Cesárea & 1 & Peridural & $\begin{array}{l}\text { Recién nacido vivo. Peso: } 3100 \\
\text { g, Apgar } 9-i \text { ? Madre desarrolló } \\
\text { cefalea post punción dural y se } \\
\text { realizaron } 2 \text { parches de sangre (el } \\
\text { segundo fue exitoso). }\end{array}$ \\
\hline $\begin{array}{l}\text { Tidmarsh }{ }^{(14)} \\
(1994-1996)\end{array}$ & Vaginal & 16 & Peridural (10) & $\begin{array}{l}\text { Seis pacientes: bloqueo sensiti- } \\
\text { vo asimétrico sin extensión sobre } \\
\text { T10. } \\
\text { Cuatro pacientes restantes: una } \\
\text { punción dural, un bloqueo asimé- } \\
\text { trico, un bloqueo excesivamen- } \\
\text { te alto y una falla en el bloqueo. } \\
\text { Conclusión de autores: analgesia } \\
\text { peridural técnicamente dificultosa } \\
\text { y con resultados imprevisibles. }\end{array}$ \\
\hline $\begin{array}{l}\text { Thompson }{ }^{(15)} \\
\text { (1999) }\end{array}$ & Cesárea & 1 & Peridural & $\begin{array}{l}\text { Recién nacido vivo. Peso } 4100 \mathrm{~g} \text {. } \\
\text { Sano. Madre no presentó eventos } \\
\text { durante hospitalización y fue dada } \\
\text { de alta al cuarto día asintomática } \\
\text { para cefalea post punción dural. }\end{array}$ \\
\hline $\begin{array}{l}\text { ASA }^{(16)} \\
(1989-2001)\end{array}$ & $\begin{array}{l}\text { Cesárea (7) } \\
\text { Vaginal (4) }\end{array}$ & $\begin{array}{c}11 \\
(8 \mathrm{EBC}+3 \mathrm{EBO})\end{array}$ & $\begin{array}{l}\text { Peridural (4) } \\
\text { Espinal (5) } \\
\text { Combinada(1) } \\
\text { General (1) }\end{array}$ & $\begin{array}{l}\text { Única complicación descrita al } \\
\text { realizar anestesia regional: ma- } \\
\text { yor número de punciones en dos } \\
\text { pacientes. Todos los bloqueos } \\
\text { fueron exitosos con anestesia sa- } \\
\text { tisfactoria. No hubo cefalea post } \\
\text { punción dural. Conclusión de au- } \\
\text { tores: anestesia regional es efec- } \\
\text { tiva y segura en pacientes obsté- } \\
\text { tricas con antecedente de espina } \\
\text { bífida. }\end{array}$ \\
\hline
\end{tabular}

\section{REFERENCIAS}

1. Kuczkowski KM. Labor analgesia for pregnant women with spina bifida: what does an obstetrician need to know? Arch Gynecol Obstet 2007;275:53-6.

2. Iglesias J, Ingilde M, Naddeo S, et al. Detección y tratamiento del mielomeningocele por un equipo interdisciplinario. Rev Hosp Mat Inf Ramón Sardá 2000;19:11-7.

3. Mitchell LE, Adzick NS, Melchionne J, Pasquariello $\mathrm{PS}$, Sutton $\mathrm{LN}$, et al. Spina bifida. Lancet 2004;364:1885-95.
4. Ali L, Stocks GM. Spina bifida, tethered cord and regional anaesthesia. Anaesthesia 2005;60:1149-50.

5. Page LK. Occult spinal dysraphism and related disorders. En: Wilkins RH, Rengachary SS, eds. Neurosurgery. New York: McGraw-Hill, 1985: 2053-7.

6. Wood GG, Jacka MJ. Spinal hematoma following spinal anesthesia in a patient with spina bifida occulta. Anesthesiology 1997;87:983-4.

7. Hunt GM, Oakeshott P. Outcome in people with open spina bifida at age 35: prospective community based cohort study. BMJ 2003;326:1365-6.

8. Rietberg CC, Lindhout D. Adult patients with spina 
bifida cystica: genetic counselling, pregnancy and delivery. Eur J Obstet Gynecol Reprod Biol 1993;52:6370.

9. Jones R. Epidural analgesia for labour in a patient with neural tube defect. Anaesthes Intensive Care 2007;35:298-9.

10. Roberts ND, May AE. Regional anaesthesia and spina bifida. Int J Obstet Anesth 2002;11:PD12. Disponible en: http://www.obstetanesthesia.com/article/S0959289X(02)80024-3/pdf

11. Piatt JH, Jr. Syringomyelia complicating myelomeningocele: review of the evidence. J Neurosurgery 2004;100(2 Suppl Pediatrics):101-9.

12. Vaagenes $P$, Fjaerestad I. Epidural block during labour in a patient with spina bifida cystica. Anaesthesia 1981;36:299-301.
13. McGrady EM, Davis AG. Spina bifida occulta and epidural anaesthesia. Anaesthesia 1988;43:867-9.

14. Tidmarsh MD, May AE. Epidural anaesthesia and neural tube defects. Int J Obstet Anesthes 1998;7:111-4.

15. Thompson MD, Vasdev GM, Findlay JY. Epidural blockade for labor and cesarean section with associated L4-5 lipomyelocele. Anesthesiology 1999;90:1217-8.

16. Stehlikova Z, Mandell GL, Golebiewski KA. Twelveyear institutional experience with obstetric anesthesia in patients with spina bifida. ASA Abstracts. Anesthesiology 2003;99:A 1175.

17. Rosaeg OP, Yarnell RW, Lindsay MP. The obstetrical anaesthesia assessment clinic: a review of six years experience. Canadian J Anaesthes 1993;40:346-56.

18. Altamimi Y, Pavy TJ. Epidural analgesia for labour in a patient with a neural tube defect. Anaesthes Intensive Care 2006;34:816-9. 